Behavior and Social Issues, 20, 122-146 (2011). (C) Gregory S. Smith, Ramona Houmanfar, \& Sushil J. Louis. Readers of this article may copy it without the copyright owner's permission, if the author and publisher are acknowledged in the copy and the copy is used for educational, not-for-profit purposes. doi: 10.5210/bsi.v20i0.3662

\title{
The Participatory Role of Verbal Behavior in an Elaborated ACCOUnt Of MEtaContingency: From CONCEPTUALIZATION TO INVESTIGATION
}

\author{
Gregory S. Smith ${ }^{1}$ \\ Ramona Houmanfar \\ Sushil J. Louis \\ University of Nevada, Reno
}

\begin{abstract}
The concept of metacontingency has been developed in order to advance an analysis of group practices and, presumably, a science of cultural change and intervention. To date discussion surrounding the metacontingency has been largely theoretical in nature. The present study arranged conditions analog to an organizational setting, in which two participants engaged in interlocking behaviors on networked computers to generate products for their hypothetical organization. Varying instructions were presented to participants throughout the experiment to determine their effect on participants' coordinated problem solving behavior. Data were collected on participants' task performance and their vocal verbal interactions as they solved problems together. The experimental conditions and data are analyzed and discussed within the framework of the metacontingency concept and thus far, the empirical data support the conceptualization of metacontingency.

KEYWORDS: metacontingency, sociology, cultural analysis, cultural selection, organizational behavior management, group practices

Behavior analysts have long made use of the behavioral contingency as a viable unit of analysis for the purposes of analyzing and subsequently modifying the behavior of individual organisms, both human and nonhuman. The elegance and effective use of this construct are at least in part due to the inductive approach through which it was derived (Skinner, 1938, 1953, 1974). Carefully replicated
\end{abstract}

\footnotetext{
${ }^{1}$ Gregory S. Smith, Department of Psychology, University of Nevada, Reno; Ramona Houmanfar, Department of Psychology, University of Nevada, Reno; Sushil J. Louis, Computer Science Department, University of Nevada, Reno. This research was supported in part by a grant from the Office of Naval Research, Department of Defense, N00014-08-1-0781. Correspondence concerning this article should be addressed to Gregory S. Smith, Department of Psychology/296, University of Nevada, Reno, Reno, Nevada 89557. E-mail: gscottsmith@unr.edu
} 
observations of behavioral phenomena were recorded and from those data principles of behavior were articulated. From these observations, Skinner (1938, 1953, 1974) derived a science of organismic behavior based on selectionistic theory; specifically, selection by consequences.

Skinner (1974) noted the revolutionary impact of a selectionist account in science, as it was first introduced by Darwin (1859) in the biological domain. In parallel, Skinner discusses a similar scientific parsimony in appealing to environmental selection at the ontogenic level, rather than some notion of transcendent inner agency. Extending the selectionist account a step further, Skinner writes of selection of a third kind: cultural selection (Skinner, 1981). The metaphor of environmental selection remained intact, yet it was expanded to account for the evolution and maintenance of practices comprising behaviors of individuals within groups.

More recently, Glenn and colleagues (Hull, Langman \& Glenn, 2001) have discussed at length the similarities between the evolution of subject matter at the biological and psychological levels of analysis (i.e., genetic and physiological characteristics of species over successive generations and behavior of individual organisms over their lifetimes, respectively). Furthermore, Glenn (1988, 2004) has speculated on the application of the selectionist approach to the third, cultural level of analysis, as Skinner had done previously. Specifically, Glenn introduced a contingency of selection to account for the organized, interlocked behaviors of multiple individuals constituting a group, known as the metacontingency.

The concept of metacontingency was derived as paralleling the behavioral contingency at the psychological level of analysis. The success of the behavioral contingency as a unit of analysis for understanding and intervening upon individual behavior presumably led to the desire for a similarly potent unit of analysis for the purpose of analyzing and intervening upon phenomena at the sociological level. Just as the behavioral contingency describes a behaviorenvironment relationship whereby classes of behavior are selected by the environmental consequences they produce, the metacontingency holds that interlocked behaviors of members constituting a group are selected by the shared environmental consequences they produce for the group members.

The metacontingency construct has undergone numerous elaborations over the last two decades. It began as roughly analogous to the two-term behavioral contingency, including lineages of interlocking behavioral contingencies (IBCs), which were regarded as the evolving entity, and the environmental demand (i.e., selecting environment; Glenn, 1988, 2004; Malott \& Glenn, 2006). Also incorporated into the original iteration of the concept was a third term, the aggregate product, produced by those individuals participating in the IBCs. 
Since its inception, others have offered elaborated accounts of the metacontingency including, most recently, up to five terms (Houmanfar, Rodrigues \& Ward, 2010). This most recent elaborated treatment of metacontingency includes the following components: cultural-organizational milieu, which represents the broader cultural and organizational context in which the phenomena of interest occur and is roughly analogous to the antecedent conditions specified in the behavioral contingency; socio-interlocked behaviors (socio-IBs), which are the organized and interlocked behaviors of group members viewed as an emergent and cohesive whole, and are held to be those higher-level entities which evolve over time as a function of the selection process; the aggregate product produced by the socio-IBs; the consumer practice, which represents the "collectivity of individuals whose behaviors and practices are not only influenced by the aggregate product, but also affect the future occurrences of the aggregate product and associated socio-IBs" (p. 90); and group-rules, which are formulated by members of the group (often leaders), in response to both the consumer practice and overarching cultural milieu and are disseminated among the group, which can then alter the function of the existing cultural-organizational milieu and hence interlocked behaviors (IBs) and emergent socio-IBs (Houmanfar et al.).

An important point in the conceptualization of metacontingency is the role of the behavior of individuals at the psychological level. Socio-IBs, described above as an emergent, cohesive unit, are in fact composed of the interlocked behaviors of organized groups of individuals. While the socio-IBs are held to be selected by factors extending into the larger environment and community, the Interlocked Behaviors (IBs; see Houmanfar et al., 2010) of one or two individuals are maintained by local contingencies of reinforcement and punishment within the organized group, through largely social and rule-governed means (Houmanfar et al., 2010). A substantial source of influence on the interlocked behaviors (IBs) of organization members arises from what may be called the cultural-organizational milieu, consisting of organizational rules, policies, and guidelines articulated and generally enforced by leaders and managers of the organization (Houmanfar et al.). Additionally, insofar as the effect of the consumer practice on the socio-IBs is mediated through consumers' interactions with the aggregate product, in response to which group-rules are generated and disseminated among group members (thus altering the cultural-organizational milieu and the psychological IBs), the selection of variations in socio-IBs is regarded as a more indirect-acting process of selection (i.e., selection is mediated through group-rules in response to consumer practices). 
Recently, researchers attempted to demonstrate the process of selection at the cultural level as described by the metacontingency construct in an analog laboratory setting (Vichi, Andery \& Glenn, 2009). The investigators employed a table-top task adapted from Wiggins (1969), in which groups of three-to-four participants worked together on a group task and contacted group consequences. Results of this study suggested that cultural selection, that is selection of the interlocked behaviors of the group members taken as a whole, occurred under the analog conditions. The work of these authors represents the first explicit attempt to experimentally observe selection at the group level, as specifically described by the metacontingency construct.

Some post-hoc descriptive analyses have been offered by proponents of the metacontingency concept (e.g., Malott \& Martinez, 2006; Todorov, 2009), yet the need for experimental analysis is warranted. Accordingly, the primary purpose of this study was to demonstrate selection of socio-cultural phenomena (i.e., socioIBs) at the group level, thereby supporting the initial findings of Vichi et al. (2009) and providing additional empirical data for analytic purposes. Although the theoretical assertion that specific behavioral patterns of individuals working together in groups and the associated aggregate product are more likely to be repeated if they produce beneficial consequences for group members is adequately analogous to operant theory, the development of metacontingency has been primarily of a deductive sort. As we see it, this stands in contrast to the inductive approach employed in the development of behavior analytic science. As such, regardless of the extent to which metacontingency conceptualization has appeared logical or adherent to common sense, we contend that empirical demonstration of theoretical concepts is necessary, in order to proceed with a scientific analysis; hence, the rationale and impetus for the present study.

Additionally, we investigated the effects of varying forms of rules presented to participants on the successful adaptation of their individual, interlocked behaviors (IBs) and emergent socio-IBs in response to varying environmental consequences (i.e., consumer practices). We argue that verbal behavior among group members and especially that which originates from influential members of the group (e.g., leaders) is a crucial component of the evolution of the sociological phenomena of interest. Therefore, characteristics of verbal behavior that may differentially influence such phenomena warrant experimental investigation. 


\section{Method}

\section{Participants, Setting, and Materials}

Participants were 20 female undergraduate students, ranging from 18 to 21 years of age, who participated for extra course credit in their respective psychology courses. In addition to earning extra course credit, participants earned monetary incentives based on their performance in the study. Participants worked together in dyads, yielding 10 dyads in all. The study was conducted in a small laboratory room on university campus, in which two desks faced each other. On each desk was a personal computer with which a participant interacted. The desks faced each other such that participants had direct visual contact with one another, but could not see each other's computer screen. The laboratory also contained an audio recording device which was used to record participants' vocal verbal interactions during the study.

\section{Independent Variables}

The independent variable manipulation consisted of the presentation of different forms of rules to participants at the beginning of each phase change. Three levels of the independent variable were employed: (A) no rules; (B) explicit rules; (C) implicit rules. The rules presented to participants at the beginning of each phase specified how participants should respond in order to correctly complete the tasks on which they worked. Explicit rules specified fairly precisely how participants should respond, while implicit rules were vague, ambiguous, and open to interpretation by participants.

\section{Dependent Measures}

The primary dependent measures were collected by the computers with which the participants interacted. Measures included frequency of correct responses and cycles (defined as production of one complete aggregate product) and time to reach solution (i.e., correct aggregate product) per condition.

\section{Experimental Design}

A counterbalanced reversal design was employed, with five dyads being exposed to each of the following two sequences of phases: ABACABACA and ACABACABA. The primary function of the reversal design was to compare the differential effects of (A) no rules, (B) explicit rules, and (C) implicit rules on 
participants' ability to effectively produce an aggregate product that met consumers' demand specifications (described in detail in procedure section). Additionally, the counterbalanced sequences were employed to assess any potential sequence effects. Carryover effects per se were not a concern, as the correct solution to the task (i.e., product meeting consumer demands) changed with each phase change, as did the independent variable manipulation (form of rule). However, we were interested to observe whether participants would produce an acceptable product during any no rule (A) condition and if so, whether this would be more likely to occur later in the session as participants had established a greater shared history of coordinating their responses.

\section{Procedure}

Participants were seated at their respective desks in the laboratory and read instructions for the experiment. The instructions advised participants that they represented two business owners of their own hypothetical company. They were told that in order to survive, their business must produce a product which had some demand in the consumer marketplace, in order to generate revenue (see Appendix A). Participants were advised that they would work together to make their hypothetical product and that they would each earn 25 cents for each correct product they produced. The researcher noted that their cumulative revenue would be tracked on screen and they would be paid whatever they had earned, in cash, at the end of the experiment. Participants then completed one practice cycle, were asked if they had any questions about how to operate the computer program, and then the experimental session began. The experimenter then left the room until the participants notified the experimenter that the study had ended.

The participants' hypothetical product comprised two parts, a rectangle and a circle. The task consisted of participants choosing both colors and sizes for the two different parts (rectangle and circle). In order to produce one complete product, it was Participant One's (P1) job to first choose the color of the rectangle portion, after which Participant Two (P2) of each dyad selected a size for that part. Upon completing the responses associated with the rectangle part, P1 then chose a size for the circle part and, lastly, P2 then chose a color for the circle part (Figure 1). Thus, four responses (two per participant), in a particular sequence, were required to complete one cycle (i.e., produce one product).

The task was sequential in nature, meaning that no one of the above responses could be emitted until the immediately prior response had been completed. In sum, the sequence for a given cycle was as follows: P1 chose color of rectangle, $\mathrm{P} 2$ chose size of rectangle, P1 chose size of circle, P2 chose color of 
circle (see Figure 1). Upon completion of all four choices, i.e., one cycle, feedback was provided to each participant on their computer screens advising whether their product met consumer specifications and earned them revenue, or whether their product did not meet consumer specifications.

The rule for determining which color and size combinations represented consumer demand for a given phase was the following: no one color, or size, could be part of the correct product in consecutive phases. In other words, if blue had been the correct color for a given phase, then any color other than blue could serve as the correct color in the subsequent phase. The same rule applied for the size property. Aside from this criterion, the correct color and size for each condition were arbitrarily preselected by the experimenter (see Appendix B for list of rules presented for each condition). In each phase this demand, in terms of color and size, was the same for both participants; in other words, the same color and size (e.g., blue and triangle-size) were correct for both participants in a given phase, as opposed to differing concurrent demands between participants.

The range of colors from which participants could choose was virtually the entire color spectrum, excluding white, black, brown, and shades of grey. However, the color solutions chosen by the experimenter were relatively simple, such as blue, green, red, orange, purple, etc. (see Appendix B). Participants selected the color for their product by manipulating a scroll bar underneath each part (rectangle and circle) on their respective computer screen. The scroll bar operated such that as the slider in the scroll bar was moved left or right, the color of the part varied along a continuum of color. For example, in the first panel of Figure 1, P1 has moved the rectangle slider to a position which produces a lightgreen color. In the fourth panel of Figure 1, P2 has moved the circle color slider slightly farther right than did P1, producing a greenish-blue color (as blue is the subsequent color on the continuum). The color chosen for the rectangle part by P1 was represented on P2's screen (Figure 1, second panel). Likewise, the color that P2 chose for the circle was displayed on P1's screen at the end of each cycle (not shown in Figure 1).

Each part of the product (rectangle or circle) was divided into five regions, which served as the size ranges from which participants could choose for their size responses. Each size range was marked with an associated arbitrary symbol (see Figure 1), such that participants could vocally reference the size they had picked or were going to pick when interacting with one another. Participants selected the size for each part of their product by manipulating a scroll bar underneath each part (rectangle and circle) on their respective computer screen. As with selecting colors, the scroll bar operated such that as the slider in the scroll bar was moved left or right, the portion (i.e., size) of the part that was filled in 
with color varied along a continuum, such that moving the slider far left resulted in none of the part being filled in, whereas moving the slider far right resulted in the part being completely filled with color. The range within which the color was filled represented the size of the part chosen by the participant (e.g., "cross" range for P1 in second panel of Figure 1, and "octagon" range for P2 in third panel of Figure 1).

The apparatus as described above operated such that as the response sliders were manipulated, the effects produced were continuous (i.e., conjugate schedule in which colors blended from one to the next and sizes increased and decreased smoothly), as opposed to discrete color and size options. In other words, it was not possible to simply choose "blue" color or "cross" size through a single, discrete option, but rather many differing response topographies along a continuum could be made, based on the precision of the participants' manipulation of the slider, that were within the functional class of producing a "blue" color or "cross" size. The cutoff range for each correct color on the color slider was selected by the experimenter, based on subjective distinctions between colors. Correct size responses consisted of the slider being manipulated such that the fill color was anywhere within the correct size region. If the color was filled exactly to one of the borders of the correct region, this was considered a correct response (although given the fine pixelation of the computer screen this was difficult to accomplish).

The duration of each phase was seven minutes, thus the duration of the entire session, consisting of nine phases, was 63 minutes. Each phase change included the presentation of one form of rule (i.e., no rule, explicit rule, or implicit rule), presented to participants on their computer screens. Phase changes were therefore signaled by the presentation of a rule when changing to a (B) or (C) condition (explicit or implicit rule, respectively); however, phase changes were unsignaled when changing to an (A) condition (no rule), as no rules appeared on the computer screen. In addition to these independent variable manipulations, each phase change consisted of a change in the consumer demand for the product (correct color and size for each part of the product). Therefore, the only means by which participants would have been aware of a change to an (A) condition would have been an instance in which they had been completing cycles correctly just prior to the phase change and, following the phase change, engaged in the same sequence of responses, thereby contacting feedback indicating an incorrect product. 
SMITH, HOUMANFAR, AND LOUIS
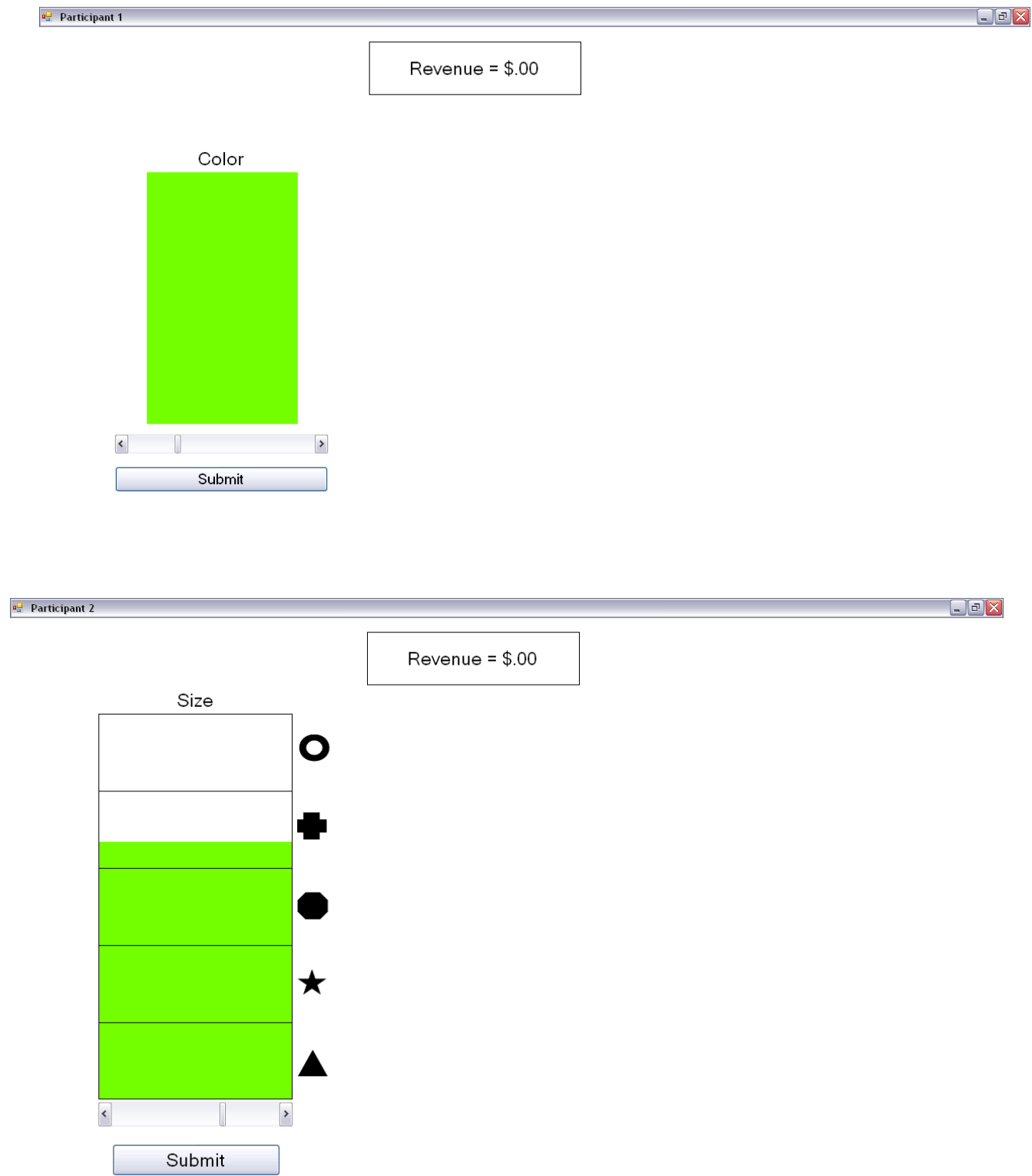

Revenue $=\$ .00$

(figure continues...) 


\section{VERBAL BEHAVIOR IN METACONTINGENCY}
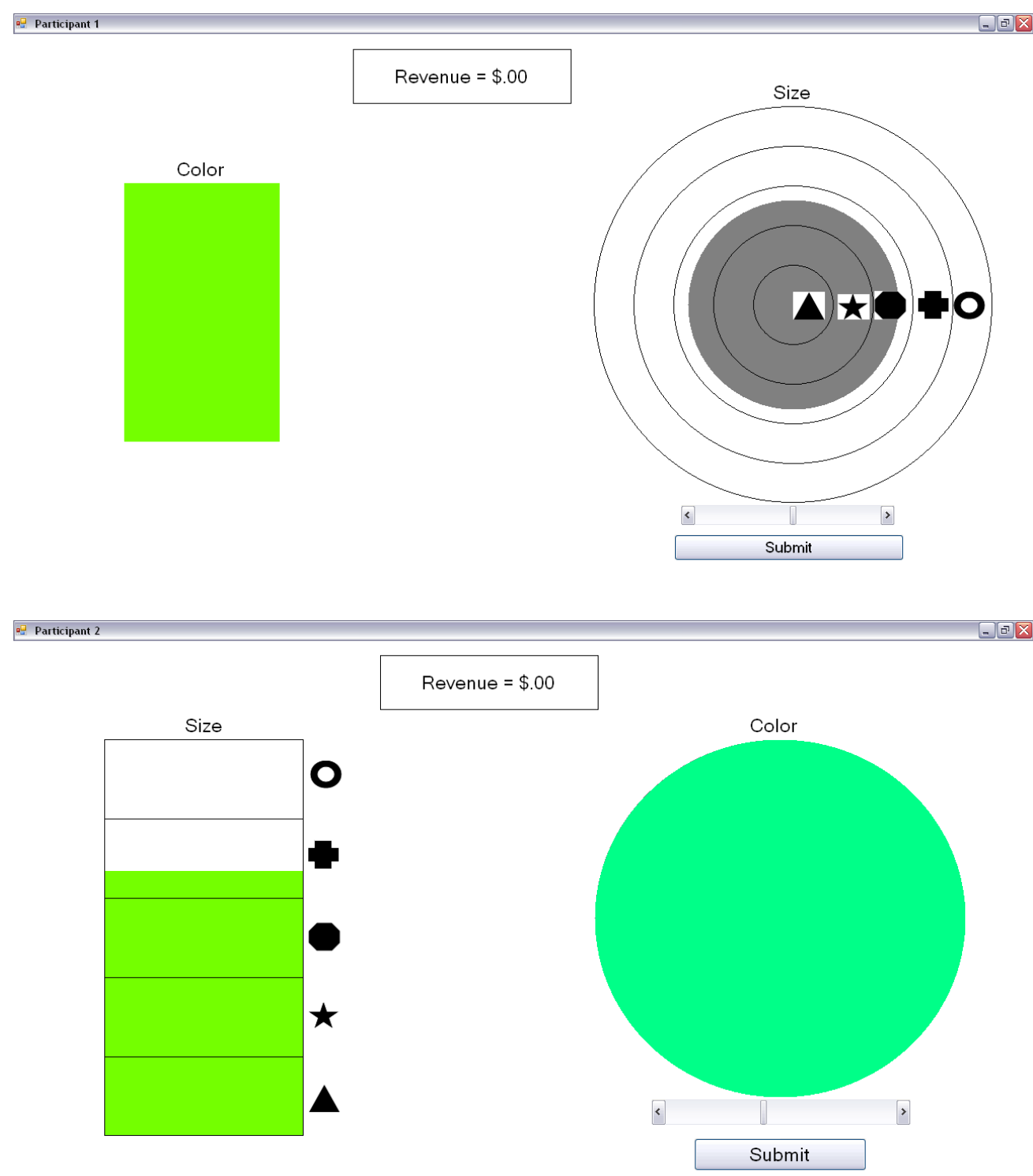

Figure 1. Screenshots of experimental apparatus. The four total responses, two per participant, are shown here in sequential order of completion. The grey color in Participant 1's circle (response 3) changed to the color chosen for the circle by Participant 2 (response 4) once that response was completed. 


\section{Results}

Data corresponding to several dependent measures are presented for several dyads in a within-subject (dyad) format. Figure 2 displays representative data for several of the dyads, some of which were exposed to the first sequence of conditions (ABACABACA, Dyads 1 and 4), and one of which was exposed to the second, counterbalanced sequence of conditions (ACABACABA, Dyad 10). In addition, Figure 3 presents data from one dyad (Dyad 5), whose performance differed from that of typical representative dyads shown in Figure 2. The figures are somewhat complex and may require elaboration.

Each panel of the figures represents data for one dyad. The abscissa for each dyad is experimental session time, presented in hours, minutes, and seconds. The ordinate axis represents the number of task responses each participant emitted correctly, per cycle. Since each cycle consisted of four total responses (two per participant), it was possible for each participant to emit zero, one, or two correct responses (represented on the ordinate axis by $0 \%, 50 \%$, and $100 \%$, respectively) per cycle. The two data streams within each panel depict each of the two participants' responses (P1 and P2) for each dyad. Data points are plotted with respect to the session time at which each cycle was completed. Thus, correct cycles (consisting of four correct responses) are indicated when the data points for each participant are both plotted on the $100 \%$ value in the figure (i.e., the solid diamond data point is inside the open square data point). The figure, then, depicts the completion of each cycle throughout the experimental session and yields information pertaining to the frequency and rate of correct cycles, the accuracy of individual responses within each cycle, the frequency and rate of cycles completed, and the temporal patterns of cycle completion, as they occurred in real-time. It is worth noting that as an artifact of the sequential nature of the task employed in the present study, each phase change was typically followed by an incorrect cycle as a result of the consumer demand changing mid-cycle. Since phase changes were determined based on a temporal criterion (seven minutes), as opposed to a fixed number of cycles completed or stability criterion, for example, it was typically the case that the phase change occurred while a particular cycle was underway, which likely resulted in the cycle being incorrect (see arrow in top panel of Figure 2). Cycles that began in one phase and terminated in the next were included, for analytical purposes, in the phase in which the cycle was completed. 


\section{VERBAL BEHAVIOR IN METACONTINGENCY}
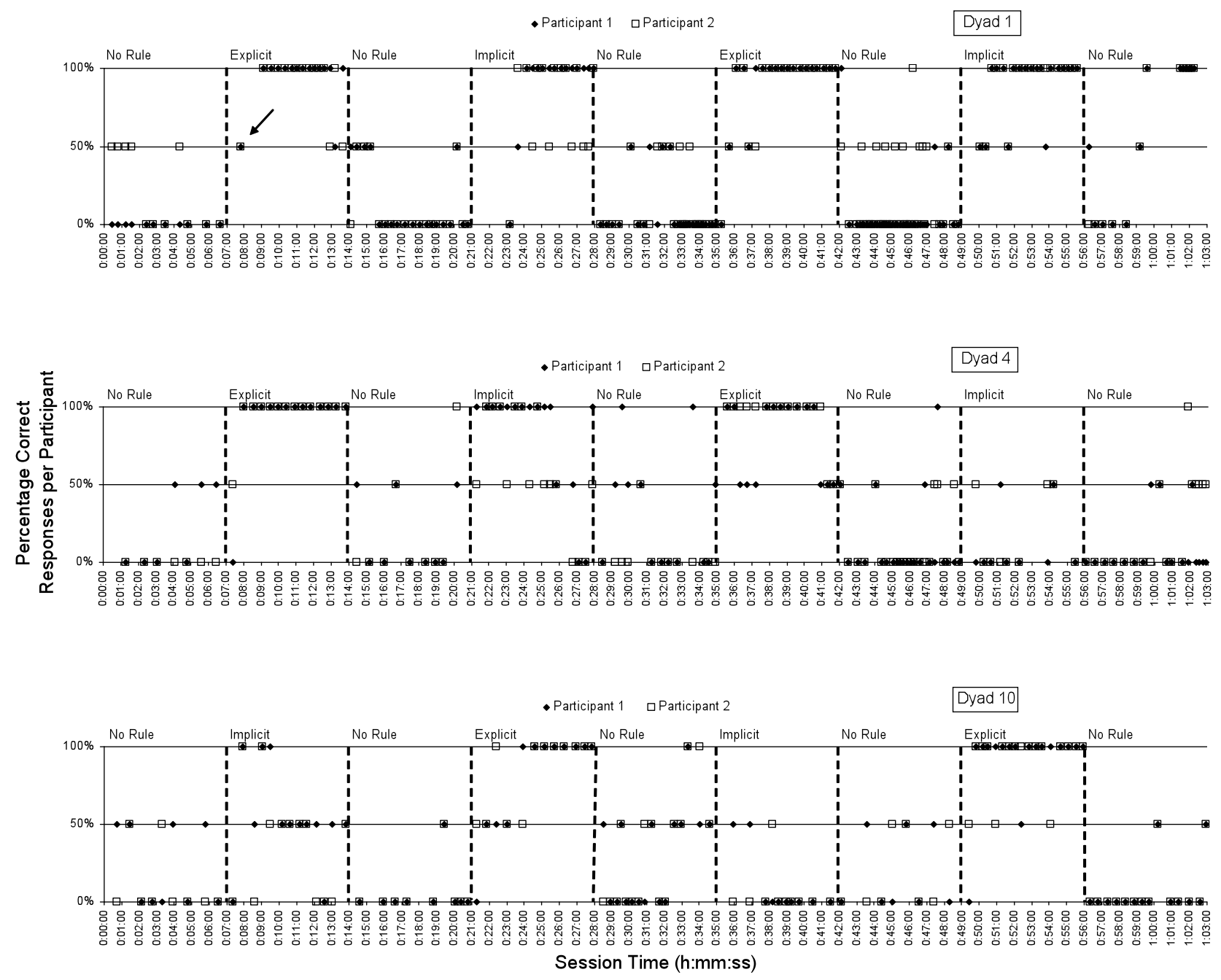

Figure 2. Real-time problem solving, per cycle, at the dyadic level. Each panel displays how many responses each participant (Participant 1 in solid diamonds, Participant 2 in open squares) completed correctly per cycle, for typical dyads (Dyads 1, 4, and 10). Data points mark the time of completion for each cycle, which consisted of four responses (two per participant), plotted against session time on the abscissa. 
Some general patterns of responding were noted across the majority of dyads (see Figure 2). For instance, most dyads completed more correct cycles during the explicit rule condition relative to the other two conditions. The exception to this was Dyad 5, which completed 17 cycles correctly during the explicit rule condition and 19 correctly during the implicit rule condition (see Figure 3). At least one cycle was completed correctly in every explicit rule phase across all ten dyads (though not every cycle completed during these phases was done so correctly). In contrast, only Dyads 1, 5, and 9 completed at least one cycle correctly in each of the two implicit rule phases to which they were exposed. Several dyads (Dyads 2, 4, 7, 8, and 10) completed at least one cycle correctly during one of the two implicit rule phases, while Dyads 3 and 6 did not complete any cycles correctly during their implicit rule exposures.

A consistent observation across all dyads was that the fewest number of cycles completed correctly occurred during the no rule condition. The single exception to this was Dyad 5, which completed 27 cycles correctly during the final no rule phase. Several dyads did not complete any cycles correctly during the no rule condition, while those dyads that did solve at least one cycle correctly did so during only one of the no rule phases (i.e., no dyad successfully completed a cycle in multiple no rule phases). A slight majority (six of ten) of dyads completed at least one cycle correctly during a no rule phase and of these, four of the six did so in the final no rule phase.

Though visual inspection of the data presented in Figures 2 and 3 yields clear differentiation of effects in relation to independent variable manipulation, statistical analyses were conducted as supplementary means of analysis. In particular, repeated measures ANOVA were run on mean correct cycles per condition and mean total cycles per condition across all dyads. These data are presented in Table 1. Total cycles completed per condition did not differ significantly based on ANOVA analysis. However, the omnibus ANOVA conducted for correct cycles did reveal statistical significance across independent variable levels, $F(2,18)=23.12, p<.001$. Follow up contrast analyses revealed that each level of independent variable was significant from each other level. Specifically, the contrast between the no rule (A) and explicit rule (B) conditions was statistically significant, $F(1,9)=41.01, p<.001$, as was the contrast between no rule (A) and implicit rule $(\mathrm{C})$ conditions, $F(1,9)=6.34, p<.05$. Lastly, the contrast between explicit (B) and implicit (C) rule conditions was also found to be statistically significant, $F(1,9)=17.37, p<.01$ (see Table 1$)$. 


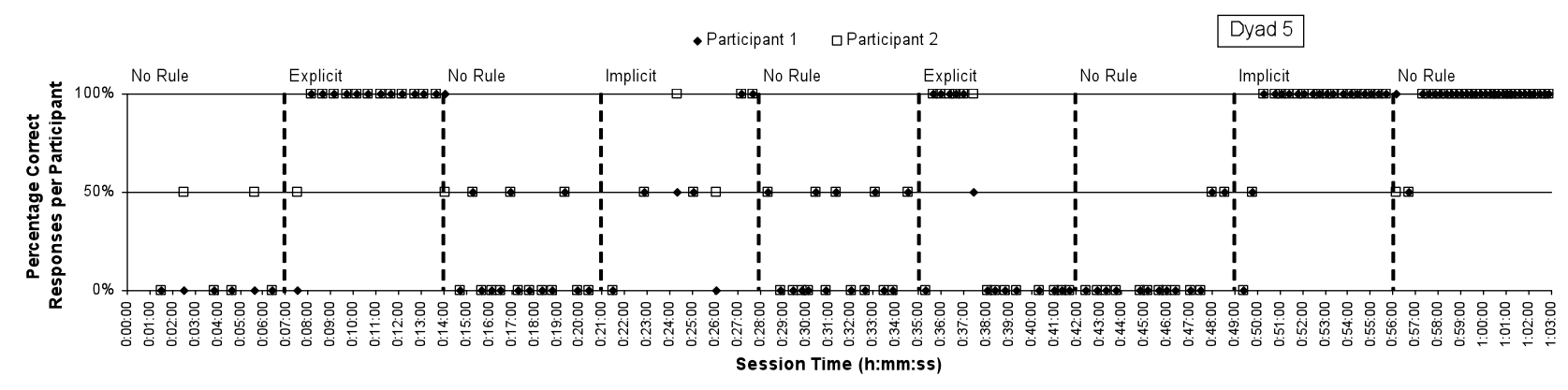

Figure 3. Real-time problem solving, per cycle, at the dyadic level. Displays how many responses each participant (Participant 1 in solid diamonds, Participant 2 in open squares) completed correctly per cycle, for atypical performance, Dyad 5. Data points mark the time of completion for each cycle, which consisted of four responses (two per participant), plotted against session time on the abscissa.

A molar (statistical mean) analysis of cycle duration per phase across dyads yielded no consistent patterns with respect to independent variable (i.e., rule) manipulation. Most, though not all, dyads exhibited somewhat of a downward trend in cycle duration over the experimental session, indicative of increased familiarity with the experimental task and a practice effect. There were, however, exceptions in which particular conditions produced longer average cycle durations. For some dyads the phases in which longer cycle durations were observed were during the explicit rule condition (Dyads 2 and 6), while for other dyads the spike in average cycle duration was observed during an implicit rule condition (Dyads 3, 4, and 5). For still other dyads, increases in average cycle duration for a given phase were observed during exposure to no rule conditions (Dyads 1, 4, 8, 9, and 10). Thus, no orderly patterns with respect to this measure emerged across all dyads in general.

Since each phase lasted exactly seven minutes, the number of cycles completed per phase is inversely related to the average cycle duration for each phase. However, the assumption that the average cycle duration for each phase accurately represents the rate of task responding for that phase masks more proximate variations in responding. A more molecular, within-dyad examination of the performance data for each dyad, as provided in Figures 2 and 3, allows for a more precise interpretation of the particular conditions which occasioned the 
SMITH, HOUMANFAR, AND LOUIS

Table 1. Mean Correct and Total Cycles per Rule Type.

\begin{tabular}{lcc}
\hline \multicolumn{1}{c}{ Type of rule } & $\begin{array}{c}\text { Correct } \\
\text { cycles }\end{array}$ & $\begin{array}{c}\text { Total } \\
\text { cycles }\end{array}$ \\
\hline No rule & $.94_{\mathrm{a}, \mathrm{c}}$ & 12.8 \\
Explicit rule & $8.7_{\mathrm{b}, \mathrm{c}}$ & 14.0 \\
Implicit rule & $3.5_{\mathrm{a}, \mathrm{b}}$ & 12.2 \\
\hline
\end{tabular}

Note. Means sharing subscript $a$ differ at $p<.05$, means sharing subscript $b$ differ at $p<.01$, means sharing subscript $c$ differ at $p<.001$, based on ANOVA comparisons.

differing patterns of responding for each dyad. For example, a review of these data indicates that a pause in task responding usually followed a signaled phase change when rules (explicit or implicit) were provided to participants. Preliminary analyses of the vocal interactions between dyadic participants indicate that these pauses in responding were typically concurrent with discussion of the provided rule, especially in the case of the delivery of implicit rules, which were ambiguous.

As seen in Figures 2 and 3, in the vast majority of instances within and across all dyads the time to reach the correct solution was fastest in the explicit rule conditions, relative to the implicit and no rule conditions. In the few instances in which dyads did reach the correct solution during a no rule condition, this typically did not occur until late within the respective phase.

\section{Discussion}

The present study empirically demonstrated that the metaphor of selection by environment is applicable at the sociological level of analysis and that sociological phenomena, termed socio-IBs (Houmanfar et al., 2010), are susceptible and sensitive to selection by environmental events (i.e., a selection contingency). The interlocked patterns of behavior of two individuals, which constituted an integrated sociological unit, were in most cases repeated when the product of that sociological unit, via its interaction with the environment (in this 
case analog consumers), produced beneficial circumstances for the group members (revenue). Although the interlocked behaviors (IBs) of the participants, which the socio-IBs comprised, were not always initially successful in producing beneficial outcomes for the group members, once they did they were typically repeated, based on that history, in order to produce the same consequences, as long as the environment supported (i.e., selected) that form of socio-IBs and aggregate product.

In addition, the present study showed that rules that take a more explicit form (relatively speaking) tend to produce greater problem-solving performance, which resulted in effective production of an aggregate product, among dyads than do more implicit rules or no rules at all. It should be noted that the distinction of explicit and implicit utilized in this study is one of form and not necessarily function (see Johnson, Houmanfar \& Smith, 2010; Pelaez \& Moreno, 1998). It cannot be said that the explicit rules utilized in the present study were entirely explicit, as they did not always produce immediately effective (correct) responding on the part of participants. In some cases, only one or two correct cycles out of many attempts were completed under the stimulus control of the explicit rules. Thus, the distinction here is one of degree, rather than kind. In other words, implicit and explicit rules as employed in the present study lie along a single continuum.

In drawing the above conclusions, several important points are worth discussing. For one, it was observed that even under conditions in which dyads were not given any rule (in terms of product color and size) as to how to respond effectively with respect to the task, some were able to coordinate their individual behaviors with one another in order to constitute an emergent sociological event which could produce a shared, beneficial outcome for the members of the group. Furthermore, when this occurred, the same sociological phenomenon typically continued to occur, in order to produce the same outcome, in the absence of any external rules to do so (i.e., selection occurred).

The importance of verbal behavior to the processes of selection and evolution at the sociological level cannot be overemphasized (Houmanfar et al., 2010). The ability of participants who constitute a dyad to coordinate their behavior, in order to discover and subsequently replicate an interlocked set of behaviors which produce beneficial outcomes, would not be possible without verbal behavior and language, especially in situations in which they were not instructed how to respond appropriately. Thus, verbal behavior between a group's individuals is not only required to initially discover a complex solution to a problem (in this case produce an aggregate product), it is also typically, though not necessarily, needed to perpetuate the occurrences of those interlocked behaviors and, more 
importantly, to facilitate and mediate coordinated variations in the interlocked behaviors (IBs), from which sociological phenomena (socio-IBS) emerge, in relation to environmental changes.

Empirical evidence from the present study illustrates the importance of verbal behavior between group members in perpetuating lineages of socio-IBs. As noted previously, vocal verbal interactions among dyads were recorded and are currently being analyzed. Preliminary analyses of these data suggest the role of these interactions in mediating the selection of socio-IBs and some examples are provided below to support this conclusion. For instance, Dyad 1 completed its first correct cycle at nine minutes and five seconds (0:09:05) into the experimental session (see Figure 2). Upon receiving feedback regarding their success, at 0:09:07 P2 in that dyad commented, "Really? So now we do it again?" to which P1 at 0:09:10 replied, "Do we keep on...? Okay, let's try it again." At 0:09:33, Dyad 1 completed its second correct cycle after engaging in the same set of socioIBs which had produced revenue on the previous cycle. Similar observations were made for Dyad 3, who at 0:08:31 completed their first successful cycle. At $0: 08: 36, \mathrm{P} 1$ in this dyad asked, "So then are we supposed to make it the same?" to which P2 at 0:08:42 replied, "Try it again." At 0:08:45, P1 commented, "They liked that orange and star, we'll try it again..." At 0:09:21, Dyad 3 completed its second successful cycle after which, at 0:09:27, P2 stated, "Just keep doing that." At 0:09:29, P1 noted, "Obviously it's working. Our customers are liking the orange and star." While these examples provide tentative support for our conclusion, further analyses are pending.

While the above observations may, at surface value, appear rather mundane and obvious, it is worth noting that without the verbal interactions among the participants that facilitated the replication of their interlocked behaviors (IBs), the observed socio-IBs may not have been repeated and therefore would not have been selected, even though they had produced a beneficial outcome for the group members. On this note, it is worth considering whether selection of the socio-IBs was actually occurring, or whether in some instances correct aggregate products were produced, but the associated socio-IBs were not actually selected and therefore did not exhibit recurring lineages as a product of environmental selection. One reason for drawing such a conclusion is that in some instances, dyads were correctly producing products during a given phase, but then during that same phase their interlocked behaviors changed and they no longer continued to produce correct products (e.g., Dyad 5, second explicit rule phase, in Figure 3).

In regard to the above concern, examination of participants' verbal interactions revealed a pattern that appeared in several dyads, based on their shared history during the experimental session. Specifically, it was found that due 
to their experience with the task, participants learned that even when they were successfully able to follow the explicit or implicit rule and produce correct products that generated revenue, the specifications that were correct at that time would eventually cease to produce products that met consumer demands (i.e., consumer demand would change). As a result, there were instances among several dyads in which a participant emitted an incorrect response during a period of successive correct cycles, which would then produce an incorrect cycle, and rather than try the previously successful responses again, they would assume that consumer demands had changed and that they therefore needed to change their responses and attempt to discover the "new" consumer demands, even though consumer demands as programmed into the computer task had not actually changed (i.e., there was no actual phase change). We believe this finding further highlights the role of verbal behavior among group members in relation to the maintenance, perpetuation, evolution, and disappearance of coordinated practices of group members, and empirically demonstrates the possibility that verbal rules may ineffectively alter interlocked behaviors, socio-IBs, and associated aggregate products with respect to prevailing environmental contingencies, as is also seen in individual psychological behavior (e.g., inaccurate tracks; see Hayes, BarnesHolmes, \& Roche, 2001).

Conceptually, these findings lend support to the elaborated account of metacontingency analysis as recently proffered by Houmanfar et al. (2010). A brief discussion of the relation between their recent five-term metacontingency elaboration and the empirical findings reported in the present study is warranted. The first of the five terms, the cultural/organizational milieu, which comprises the context in which the socio-IBs occur and consists of, among other things, the needs of the group and its members, the resources available in the environment, any form of competition, and verbal rules pertaining to individuals' behavior, has been sufficiently addressed in Houmanfar \& Rodrigues (2006) and in Houmanfar et al. (2010) and will not be discussed further here.

The second term in Houmanfar et al.'s (2010) five-term metacontingency is the socio-IBs, which is regarded as a sociological phenomenon and which is held to be emergent (i.e., substantively different) from the psychological behaviors of which it is composed. In the present study, the socio-IBs are represented by the set of interlocked behaviors of participants in a dyad which produced an aggregate product. The third term is the aggregate product itself, which is typically (and in this case was) that which interacts with the selecting environment external to the group. In this particular study, the aggregate product consisted of a hypothetical product made up of circle and rectangle parts that were assigned certain properties (color and size) by participants. The aggregate product could not have been com- 
pleted by any one participant in this case, given the interlocked and sequential nature of the responses required for its production.

The fourth term of the proposed five-term metacontingency unit is the consumer practice. In the present study, the consumer practice was simulated analogously and was predetermined, in terms of product characteristics demanded by consumers, based on the experimenter's arbitrary selection of "correct" product colors and sizes. The fifth term in the metacontingency has been termed "group-rule generation." Houmanfar et al. (2010) define this term as, "the cultural entity's (e.g., organization) response to the practices of consumers or the public, typically in the form of a group-rule crafted by those in power that may alter the cultural milieu and the socio-IBs," (p. 90). Further, the authors suggest that selection of the socio-IBs that produced the aggregate product is often an indirect process, involving verbal behavior and language as an intermediary. In other words, consumers interact with the aggregate product directly, but do not often interact directly with the socio-IBs, as a cohesive whole, which produced the aggregate product. Thus, the selection of the socio-IBs is typically dependent upon rule-governance, resulting from the generation of rule statements by members who are usually, but not necessarily, leaders of some sort within the organization. The rule statements generated by these group members are, as Houmanfar et al. explain, in response to the consumer practice.

Some common data pertaining to consumer practices that provide feedback to organizations and influence group-rule generation are sales reports, consumer ratings and surveys, focus groups, and customer service/complaint logs. In the present study, the feedback to the dyadic organizations consisted of a statement following the completion of each product (cycle), which informed organization members whether or not their product met consumer demands. In addition, under some conditions dyads were presented with rule statements from the experimenter, which may be conceptualized as group-rules presented by an authority within the organization (e.g., manager, executive officer).

Group-rule statements may be codified into formal organizational rules, policies, procedures, or job descriptions, and it is typically in this manner that the interlocked, psychological behaviors of many individuals (usually employees) are quickly and efficiently modified. As such, these evolving group-rule statements become a part of the existing organizational milieu, which is thus transformed in terms of form and function and sets the occasion for the occurrence of the IBs and hence the emergent socio-IBs. It should be noted, however, that the group-rule generation need not always be on the part of organizational leaders. In the present study, the organizational members were hierarchically parallel in terms of their 
official position within and presumed influence over the organization (i.e., neither member of a dyad was formally a leader nor subordinate).

As the participants generated rule statements with respect to the experimental setting and task, these rules influenced both their individual psychological behavior (IBs) and the emergent socio-IBs as a unitary whole, in addition to the aggregate product produced by the socio-IBs. In essence, the participants were generating group-rules which, once uttered, and further depending upon their functional effects on one another, became part of their organizational milieu and hence influenced and altered the socio-IBs and aggregate product. Furthermore, in circumstances in which dyads had discovered and successfully replicated a correct solution that produced a beneficial outcome for the dyad (i.e., revenue generation), once the dyad was exposed to changing environmental (consumer) demands the verbal behavior on the part of participants, especially in the form of group-rules, which influenced both participants' behavior in functionally similar ways, was the only means by which participants were able to systematically alter their interlocked behaviors (IBs) and thus the socio-IBs and successfully produce and replicate a new aggregate product.

This study has provided empirical evidence for not only the general assumption that selection of sociological entities (socio-IBs) does indeed occur, but it has also provided means for analyzing some of the processes through which selection and evolution at this level of analysis proceed. Based on the empirical observations made in the present study, the elaboration of the five-term metacontingency, as a unit of analysis used to describe the evolution of sociological entities, appears to describe the relationships among the observed sociological phenomena in a coherent and parsimonious manner. Furthermore, this line of reasoning and investigation bears implications for the design and implementation of interventions intended to achieve sociological effects. Specifically, psychological events (e.g., behavioral interventions) that influence the behavior of group members who can in turn influence group-rule generation (typically by leaders) can ultimately have sociological effects, by means of influencing the interlocked behaviors (IBs) of the individuals of which the organization is composed, the socio-IBs which are emergent upon those interlocked behaviors, and the aggregate product of the socio-IBs (see Houmanfar, Rodrigues, \& Smith, 2009, and Ward, 2009 for further discussion).

Future research in this and relevant lines should take into consideration several limitations associated with the present study. One such limitation regards the nature of the analog task that was utilized. A more realistic (i.e., high-fidelity) and possibly more complex work task may better approximate conditions found in the naturalistic settings with which we are interested. Caution must be taken to 
avoid making the task too complex, however, such that the phenomena of interest may be occasioned and observed in the temporally condensed analog setting. Another limitation of the present study was the duration-based criterion utilized to control phase changes. As a result of this procedure considerable variability was found in the pace and overall frequency of cycle completions across dyads (though these differences did not differ significantly based on statistical analyses). Ideally, a different criterion such as a performance-based stability criterion could be employed to dictate if or when phase changes, including changing consumer demands, should occur. Additionally, given the nature of the interlocked, sequential responses in which participants had to engage to complete each cycle, the problem of a phase change occurring mid-cycle was common and, depending upon the sequence of the phase change, typically resulted in an incorrect cycle.

Yet another aspect of the present study that could be considered a potential limitation, but was of particular interest in the present research, was the cumulative effect of the shared history of interaction that was built up between dyad members throughout the experimental session. In most instances in which dyads effectively problem solved and produced a correct product during a no rule condition, it was found to have occurred in one of the latter no rule phases. This pattern is possibly indicative of more effectively coordinated interlocked behaviors among participants resulting from their experimental history of working together. Thus, attempts to control for this learning as it occurs during the experimental session may be desirable, depending upon the research question, though it is difficult to imagine how one might control for such a variable.

The experimental framework and apparatus developed for the present study provides numerous opportunities for future research. For example, one such study could involve examining the effects on problem solving performance and verbal interactions of successive generations of participants within each dyad (see Baum, Richerson, Efferson \& Paciotti, 2004). This would allow observations to be made pertaining to the processes through which individuals are indoctrinated into organized groups (socio-IBs) and come to replace others with respect to specific roles within the group. Additional research could focus on manipulating the feedback component of the procedure; specifically, feedback could be more or less descriptive or corrective, it could be presented on various schedules, and it could be presented with respect to only one participant's behavior at a time or to only one participant at a time.

Another potential study could investigate the effects of differing shared learning histories of dyad members on their cumulative problem solving repertoires. For instance, one could ask whether a shared history in which only explicit rules have been provided to dyad members as they work together 
produces a more or less adaptive repertoire (i.e., generation of effective grouprules) in the presence of changing environmental demands (consumer practice), relative to dyads receiving only implicit rules, no rules, or any particular sequence of rule types. As discussed in Hayes, Barlow, and Nelson-Gray (1999, p. 189), alternation effects, a subset of carryover effects, can have an impact on responding, based on the schedule of alternation of conditions; in essence, the design itself becomes an independent variable. In addition, resource requirements could be placed on dyads, such that organizational resources are depleted over time (e.g., expenses), forcing dyad members to quickly learn to respond effectively in order to maintain sufficient resources for their organization's survival. Lastly, future research could incorporate more than two participants per group and could add complexity through the delineation of hierarchical roles (e.g., leaders) within groups.

It should be noted that the authors are currently working on extending the present study by coding and analyzing the recorded vocal interaction data between dyad members. Specifically, we are analyzing the patterns of vocal interaction as occasioned by the various rule conditions, as well as determining any correlations among interaction patterns and problem solving performance (correct aggregate product) measures. The interpretations and conclusions drawn from this work will inform ongoing research of the authors' pertaining to the investigation of psychological and sociological processes involved in more complex cultural entities. In particular, crews of naval officers in command of Navy warships will be investigated in terms of the interlocked behaviors of their members (roles and duties), the emergent socio-IBs which consist of their cohesive group functioning (tactical decision making), and the role of verbal behavior (team communication) in producing their aggregate product and associated outcomes.

\section{References}

Baum, W. M., Richerson, P. J., Efferson, C. M., \& Paciotti, B. M. (2004). Cultural evolution in laboratory microsocieties including traditions of rule giving and rule following. Evolution and Human Behavior, 25, 305-326.

Darwin, C. (1859). On the origin of species. London: John Murray.

Glenn, S. S. (1988). Contingencies and metacontingencies: Toward a synthesis of behavior analysis and cultural materialism. The Behavior Analyst, 11, 161-179.

Glenn, S. S. (2004). Individual behavior, culture, and social change. The Behavior Analyst, 27, 133-151.

Hayes, S. C., Barlow, D. H., \& Nelson-Gray, R. O. (1999). The scientist practitioner, $2^{\text {nd }} e d$. Needham Heights, MA: Allyn \& Bacon. 


\section{SMITH, HOUMANFAR, AND LOUIS}

Hayes, S. C., Barnes-Holmes, D., \& Roche, B. (2001). Relational Frame Theory: A postSkinnerian account of human language and cognition. New York, NY: Kluwer Academic/Plenum Publishers.

Houmanfar, R., \& Rodrigues, N. J. (2006). The metacontingency and the behavioral contingency: Points of contact and departure. Behavior and Social Issues, 15, 13-30.

Houmanfar, R., Rodrigues, N. J., \& Smith, G. S. (2009). Role of communication networks in behavioral systems analysis. Journal of Organizational Behavior Management, 29(3-4), 257275.

Houmanfar, R., Rodrigues, N. J., \& Ward, T. A. (2010). Emergence and metacontingency: Points of contact and departure. Behavior and Social Issues, 19, 78-103.

Hull, D. L., Langman, R. E., Glenn, S. S. (2001). A general account of selection: Biology, immunology, and behavior. Behavioral and Brain Sciences, 24, 511-573.

Johnson, R. A., Houmanfar, R., \& Smith, G. S. (2010). The effect of implicit and explicit rules on customer greeting and productivity in a retail organization. Journal of Organizational Behavior Management, 30, 38-48.

Malott, M. E., \& Glenn, S. S. (2006). Targets of intervention in cultural and behavrioal change. Behavior and Social Issues, 15, 31-56.

Malott, M. E., \& Martinez, W. S. (2006). Addressing organizational complexity: A behavioural systems analysis application to higher education. International Journal of Psychology, 41, 112.

Pelaez, M., \& Moreno, R. (1998). A taxonomy of rules and their correspondence to rule-governed behavior. Mexican Journal of Behavior Analysis, 24 (2), 197-214.

Skinner, B. F. (1938). Behavior of organisms: An experimental analysis. Cambridge, MA: B. F. Skinner Foundation.

Skinner, B. F. (1953). Science and human behavior. New York: Free Press.

Skinner, B. F. (1974). About behaviorism. New York: Vintage.

Skinner, B. F. (1981). Selection by consequences. Science, 213, 501-504.

Todorov, J. C. (2009). Behavioral analysis of non-experimental data associated with cultural practices. Behavior and Social Issues, 18, 10-14.

Vichi, C., Andery, M. A. P. A., \& Glenn, S. S. (2009). A metacontingency experiment: The effects of contingent consequences on patterns of interlocking contingencies of reinforcement. Behavior and Social Issues, 18, 41-57.

Ward, T. A. (2009). Piracy in Somalia: Interbehavioral assessment and intervention. Behavior and Social Issues, 18, 136-154.

Wiggins, J. A. (1969). Status differentiation, external consequences, and alternative reward distributions. In R.L. Burgess, \& D. Bushell, Jr. (Eds.), Behavioral sociology: The experimental analysis of social processes. New York: Columbia University Press, pp. 109126. 


\section{Appendix A}

\section{Instructions Read to Participants}

"You two represent two business owners in charge of a small company. In order to survive, your company must make a product which has some demand in the consumer marketplace. The two of you will work together to make your product, in order to generate revenue for your company. You will receive feedback after every product you make informing you whether it met consumers' demands or not. For every product you make that meets consumers' demands, you will earn revenue in the amount of 25 cents each, which will be tracked on screen for you. Any revenue you earn during the study is yours to keep upon completion of the study, so you should try to make as many products correctly as you can, in order to maximize your revenue.

"Periodically throughout the study you will see instructions pop up on the screen which will give you a hint as to how you should produce your product, in terms of size and/or color. These hints, when they appear, are accurate with respect to what the consumers want. In addition, as in the real world, what consumers want may change over time.

"Your verbal interactions with one another will be recorded throughout the study. You will be left alone in this room until the computer program stops, at which time a message will appear alerting you that the study is done. At that time I will return and stop audio recording. I will provide a debriefing of the study, as well as pay you your revenues in cash.

"Do you have any questions at this time?

"I will now demonstrate for each of you how to use the computer program to make your products." 


\section{Appendix B}

\section{Consumer Demands and Rule Statements}

\begin{tabular}{|c|c|c|}
\hline Color & Size & Rule Statement \\
\hline Blue & Cross & \\
\hline Orange & Star & $\begin{array}{l}\text { "Your product must be ORANGE in color and } \\
\text { within STAR size." }\end{array}$ \\
\hline Red & Triangle & \\
\hline Yellow & Circle & $\begin{array}{l}\text { "Your product must be the lightest primary color } \\
\text { and large in size." }\end{array}$ \\
\hline Purple & Octagon & \\
\hline Pink & Triangle & $\begin{array}{l}\text { "Your product must be PINK in color and within } \\
\text { TRIANGLE size range." }\end{array}$ \\
\hline Green & Cross & \\
\hline Light Blue & Star & $\begin{array}{l}\text { "Your product must be a light shade of the fifth } \\
\text { color of the rainbow and a smaller size." }\end{array}$ \\
\hline Red & Circle & \\
\hline
\end{tabular}

